\title{
The Appointment, Composition, Design Features \\ and Characteristics of the Ground Equipment \\ for Registration Radar Signals \\ in the Centimeter Wavelength Range
}

\author{
Aleksandr A. Antsiferov*a, Aleksandr V. Bogdanov \\ Viktor N. Bondarev ${ }^{\mathrm{b}}$, Denis V. Zakomoldin ${ }^{\mathrm{a}}$, \\ Ivan M. Ivanova, Aleksandr A. Kuchin ${ }^{\mathrm{a}}$, \\ Valery M. Belyaev ${ }^{c}$, Valentin A. Golubenko ${ }^{c}$, \\ Maksim V. Dedov', Valery V. Drogalin', \\ Evgeny S. Ivanov', Nikita A. Shestakov', \\ Igor V. Lyutikov*b and Valery N. Tyapkin ${ }^{\mathrm{b}}$ \\ ${ }^{a}$ Military Academy of Aero-Space Defence \\ named after the Marshal of Soviet Union G.K. Zhukov \\ 50 Zhigareva Str., Tver, 170022, Russia \\ ${ }^{b}$ Siberian Federal University \\ 79 Svobodny, Krasnoyarsk, 660041, Russia \\ 'Joint-stock company \\ Research and Production Complex «PELENGATOR》 \\ 31-33 Ivan Chernykh Str., St. Petersburg, 198095, Russia
}

Received 14.12.2017, received in revised form 04.02.2018, accepted 12.03.2018

The article discusses the purpose, composition, design features and characteristics of the land instrument registration of radar signals in the centimeter wavelength range and its main components.

Keywords: radar signal recording equipment, centimeter wavelength range, statistical characteristics of radar signals.

Citation: Antsiferov A.A., Bogdanov A.V., Bondarev V.N., Zakomoldin D.V., Ivanov I.M., Kuchin A.A., Belyaev V.M., Golubenko V.A., Dedov M.V., Drogalin V.V., Ivanov E.S., Shestakov N.A., Lyutikov I.V., Tyapkin V.N. The appointment, composition, design features and characteristics of the ground equipment for registration radar signals in the centimeter wavelength range, J. Sib. Fed. Univ. Eng. technol., 2018, 11(3), 318-324. DOI: 10.17516/1999-494X-0044.

(C) Siberian Federal University. All rights reserved

* Corresponding author E-mail address: antsiferovalexandr@gmail.com, lyutikovigor@mail.ru 


\section{Назначение, состав, конструктивные особенности}

и характеристики наземной аппаратуры

регистрации радиолокационных сигналов

в сантиметровом диапазоне волн

А.А. Анциферов ${ }^{\mathrm{a}}$, А.В. Богданов ${ }^{\mathrm{a}}$, В.Н. Бондарев ${ }^{\sigma}$, Д.В. Закомолдин ${ }^{\text {a }}$, И.М. Иванов ${ }^{a}$, А.А. Кучин ${ }^{\mathrm{a}}$, В.М. Беляев ${ }^{\mathrm{B}}$, В.А. Голубенко М.В. Дедов ${ }^{\text {в }}$ В.В. Дрогалин ${ }^{\text {, }}$ Е.С. Иванов ${ }^{\text {, }}$ Н.А. Шестаков ${ }^{\text {, И.В. Лютиков }}{ }^{6}$, В.Н. Тяпкин ${ }^{6}$ ${ }^{a}$ Военная академия воздушно-космической обороны им. Маршала Советского Союза Г.К. Жукова Россия, 170022, Тверь, ул. Жигарева, 50 ${ }^{6}$ Сибирский федеральный университет Россия, 660041, Красноярск, пр. Свободный, 79 ${ }^{8}$ Акиионерное общество «Научно-производственный комплекс «ПЕЛЕНГАТОР» Россия, 198095, Санкт-Петербург, ул. Ивана Черных, 31-33

B статье рассматривается назначение, состав, конструктивные особенности и характеристики наземной аппаратуры регистраџии радиолокаџионных сигналов в сантиметровом диапазоне волн и её основных узлов.

Ключевые слова: аппаратура регистрачии радиолокационных сигналов, сантиметровый диапазон волн, статистические характеристики радиолокационных сигналов.

С целью создания банка данных спектральных и траекторных статистических характеристик радиолокационных (РЛ) сигналов, отражённых от различных классов и типов реальных воздушных целей, в интересах формирования экспериментальных исходных данных для разработки алгоритмов распознавания целей в РЛ-системах наземного базирования была разработана аппаратура регистрации РЛ-сигналов в сантиметровом диапазоне волн.

Целью статьи является общая характеристика данной аппаратуры, включающая её назначение, состав, конструктивные особенности и тактико-технические характеристики.

\section{Назначение}

Аппаратура предназначена для регистрации в реальном масштабе времени в сантиметровом диапазоне волн РЛ-сигналов, отражённых от реальных воздушных объектов.

Состав аппаратуры

1. Ноутбук с программно-алгоритмическим обеспечением (ПАО), разработанным на предприятии АО «НПК ПЕЛЕНГАТОР».

2. Блок формирования и обработки сигнала (БФСО), разработанный на предприятии АО «НПК ПЕЛЕНГАТОР». 
3. Антенное устройство (АУ), разработанное на предприятии АО «НПК ПЕЛЕНГАТОР».

4. Бесплатформенная инерциальная навигационная система (БИНС), разработанная на предприятии ООО «ТеКнол».

5. Универсальный контейнер (УК), разработанный на предприятии АО «НПК ПЕЛЕНГАTOP».

6. Устройство охлаждения (УО), разработанное на предприятии ООО «Криотерм».

Как завершённое изделие аппаратура имеет вид, представленный на рис. 1, на котором обозначены 1 - АУ, 2 - БФСО, 3 - УО, 4 - БИНС, 5 - ноутбук с ПАО.

Основные характеристики аппаратуры регистрации:

эффективная изотропно излучаемая мощность - не менее 44,5 дБ/Вт;

энергетическая добротность АУ в режиме «приём»- не менее минус 30 дБ/К;

коэффициент усиления антенны в режиме «приём»- не менее 28,5 дБ;

дальность действия - не менее 15 км;

длительность излучаемых импульсов - $1 \pm 0,1$ мкс;

коэффициент шума приёмного тракта - не более 4;

ширина диаграммы направленности антенны (ДНА) в азимутальной плоскости (рис. 2) по уровню минус ЗдБ - не более 7 град.;

ширина ДНА в угломестной плоскости (рис. 3) по уровню минус ЗдБ - не более 8 град.;

относительный уровень боковых лепестков - не более минус 23 дБ;

рабочий сектор электронного сканирования при условии отсутствия побочных дифракционных максимумов:

- в азимутальной плоскости: $\pm 45^{\circ}$;

- в угломестной плоскости: $\pm 30^{\circ}$.

В результате натурных экспериментов аппаратура регистрации успешно прошла испытания и полученные реальные её характеристики достаточно хорошо сходятся с заявленными теоретическими.

Основу антенного устройства (рис. 4) составляет комбинированная активная фазированная антенная решётка (КАФАР).

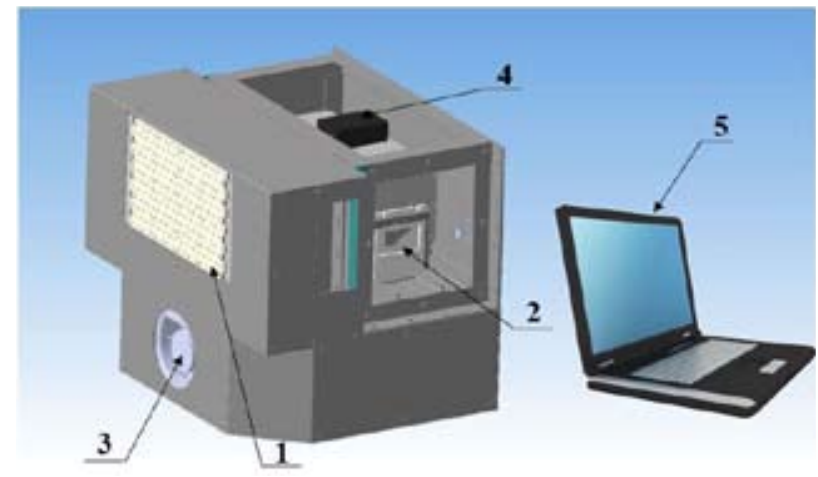

Рис. 1. Конструкция аппаратуры

Fig. 1. Construction of equipment 


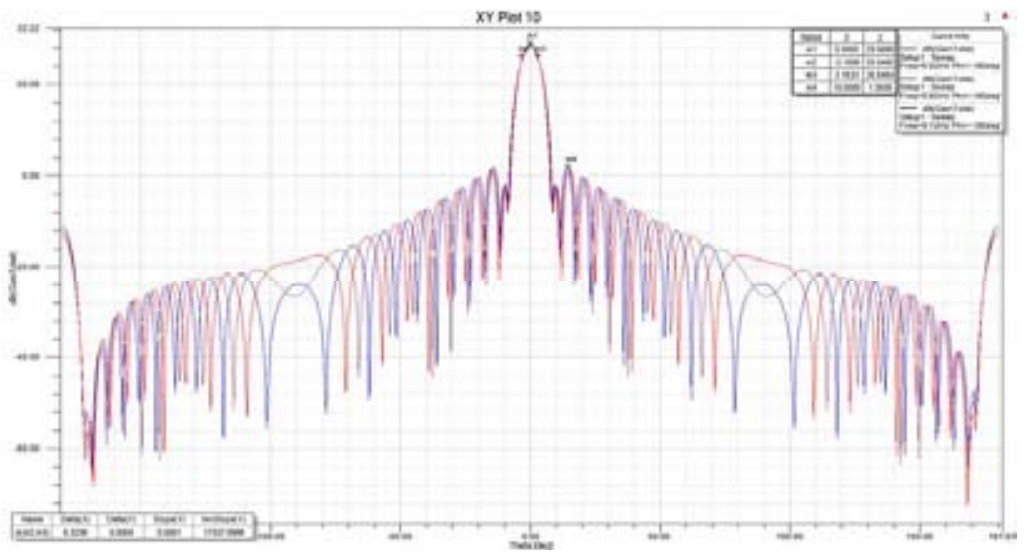

Рис. 2. Сечение ДНА-антенны в азимутальной плоскости

Fig. 2. Cross-section of the antenna pattern in the azimuth plane

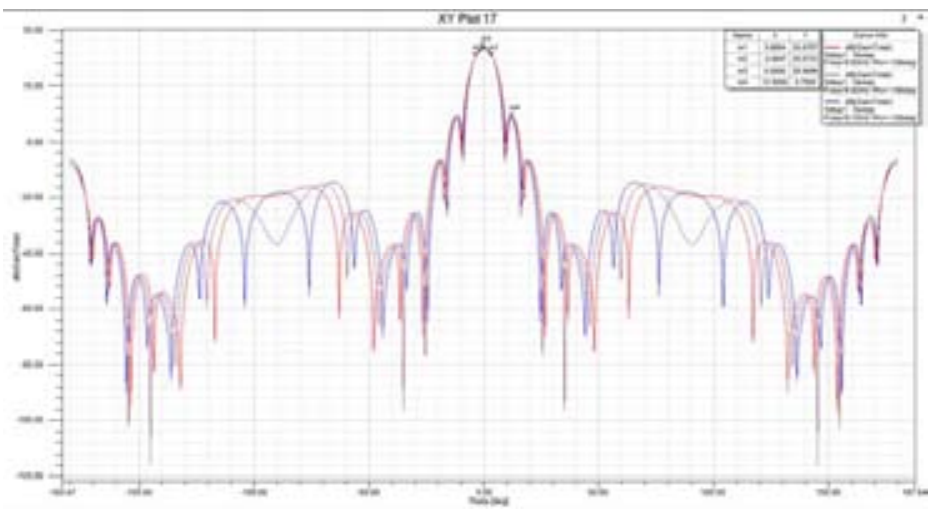

Рис. 3. Сечение ДНА-антенны в угломестной плоскости

Fig. 3. Cross-section of the antenna pattern in the vertical plane

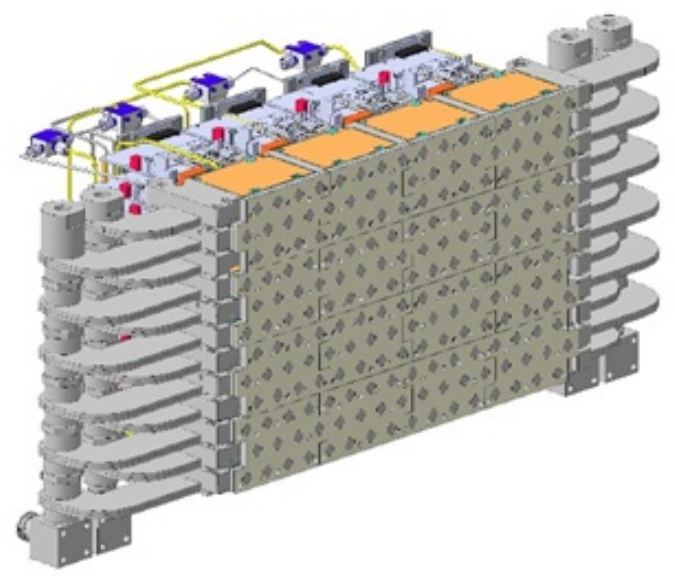

Рис. 4. Конструкция антенного устройства

Fig. 4. Antenna device design 
В состав АУ входит:

36 приёмных модулей;

12 приёмопередающих модулей, расположенных в центре антенного полотна;

16 модулей антенного полотна (МАП);

антенна пилот-сигнала;

высокочастотная распределительная система (сумматоры 3:1 и сумматоры 2:1);

низкочастотная распределительная система;

система управления лучом.

Каждый из 16 МАП имеет вид, представленный на рис. 5.

В состав одного МАП входят 12 излучателей. Конструктивно он выполнен из двухслойной микрополосковой структуры. На поверхности слоя 1 методом травления выполнены металлизированные излучатели (резонаторы), гальванически не связанные с директорными излучателями, нанесёнными на слой 2 диэлектрической подложки.

Каждый и 12 приёмопередающих модулей (MMTR8591-40) состоит из четырех отдельных модулей приёма/передачи (рис. 6).

Четыре канала приёмопередающего модуля могут работать как одновременно, так и независимо друг от друга. Каждый канал состоит из дискретного фазовращателя, дискретного аттенюатора, переключателя, усилителя средней мощности, усилителя высокой мощ-
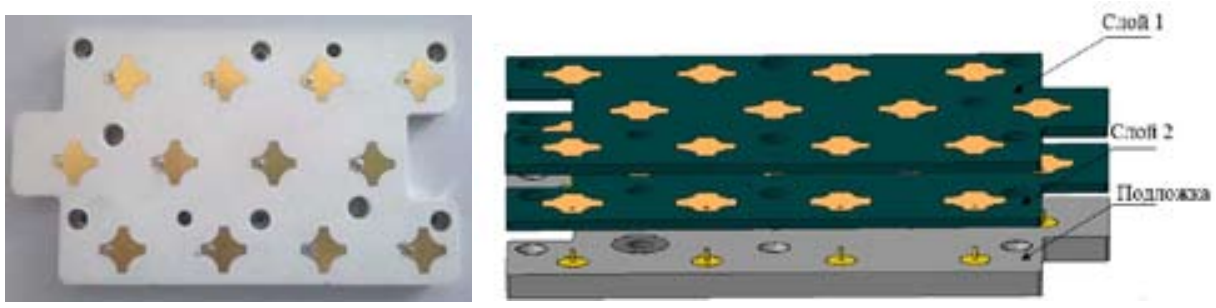

Рис. 5. Модуль антенного полотна

Fig. 5. Module of antenna curtain

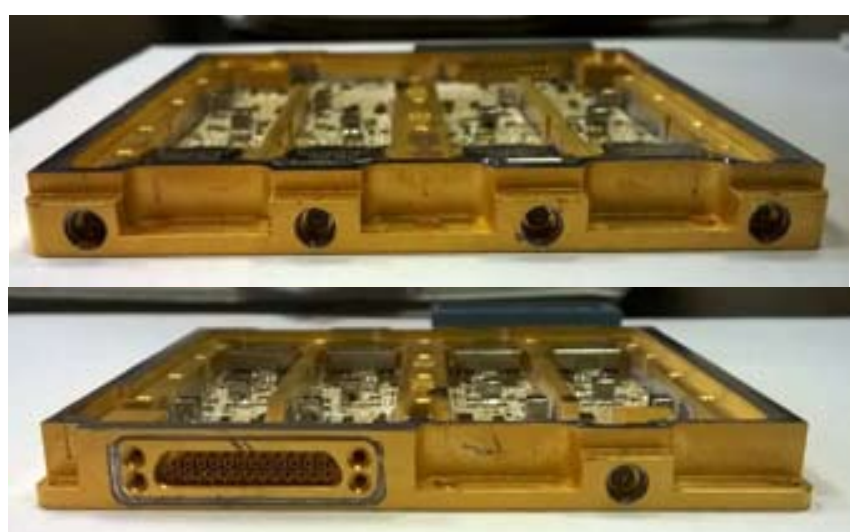

Рис. 6. Приёмопередающий модуль MMTR8591-40

Fig. 6. Receiving-transmitting module MMTR8591-40 
ности, циркулятора, ограничителя, усилителя с низким уровнем шума, сигнальных схем и др.

Устройство охлаждения (рис. 7) предназначено для поддержания теплового режима аппаратуры регистрации при проведении экспериментов в течение 6 ч непрерывной работы в диапазоне температур окружающей среды от минус 40 до плюс $70{ }^{\circ} \mathrm{C}$.

В состав конструкции жидкостных радиаторов АУ (рис. 8) входят:

входные патрубки (1);

радиаторы охлаждения приёмопередающего модуля (2);

коллектор нечетных радиаторов (3);

коллектор чётных радиаторов (4);

выходные патрубки (5).

Блок формирования сигналов и обработки (рис. 9) является малогабаритным многофункциональным радиотехническим устройством, предназначенным для приёма и передачи СВЧ-сигнала Х-диапазона, преобразования на промежуточную частоту, оцифровки его много-

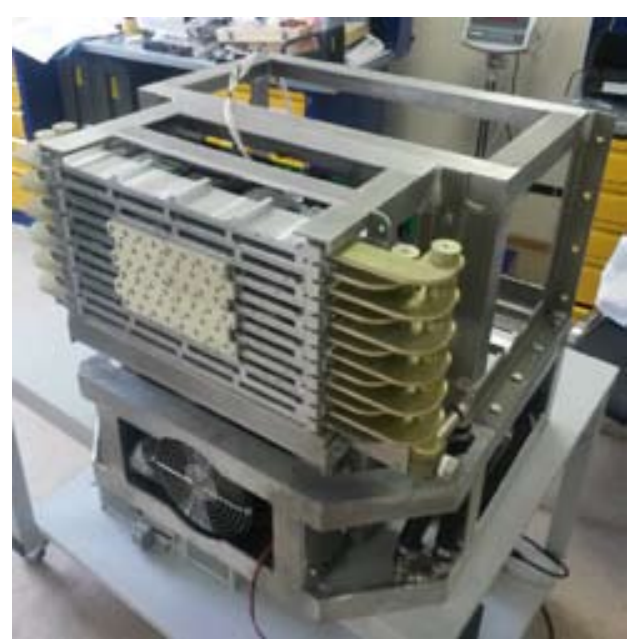

Рис. 7. Устройство охлаждения

Fig. 7. Cooling device

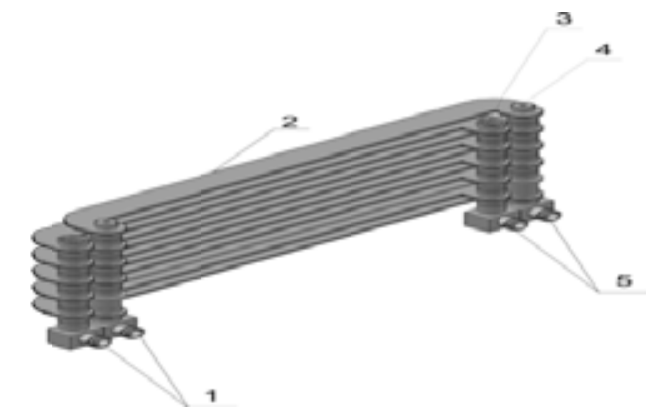

Рис. 8. Модель конструкции радиаторов АУ

Fig. 8. Model of construction of radiators of the antenna device 


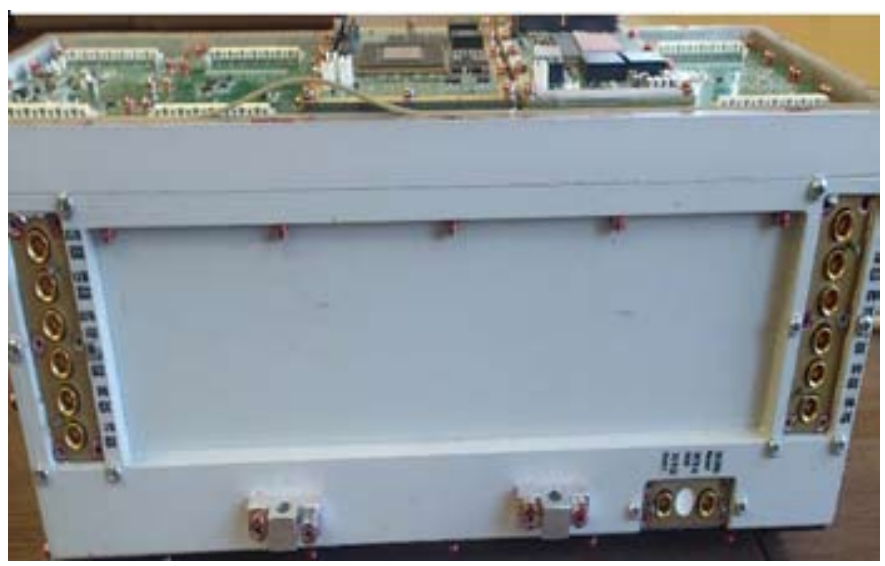

Рис. 9. БФСО

Fig. 9. Reference signal generation unit

канальным аналого-цифровым преобразователем, многоканальной цифровой обработки сигнала в блоке цифровой обработки и передачи результатов обработки через модуль интерфейсов.

В состав БФСО входят: генератор частот; синтезатор частот; модуль цифровой обработки; панель коммутационная; модуль интерфейсов; высокочастотные модули RRF; плата SOFury; корпус; программное обеспечение БФСО.

Таким образом, определены назначение, состав, конструктивные особенности и характеристики наземной аппаратуры регистрации радиолокационных сигналов в сантиметровом диапазоне волн. 